\title{
MODIFIED BOUNDARY INTEGRAL METHOD FOR PRESSURE DAIVEN MHD DUCT FLOW
}

\author{
B.D. AGGARWALA and P.D. ARIEL \\ Department of Mathematics and Statistics \\ University of Calgary, \\ Calgary, Alberta, Canada
}

(Received January 15, 1988)

ABSTRACT. In this paper, we investigate the flow of a viscous, incompressible, electrically conducting fluid through a rectangular duct in the presence of a magnetic field, when one of the boundaries perpedicular to the magnetic field is partly conducting and partly insulating, by a modifled Boundary Integral Method.

Three problems are considered (1) flow through an infinite channel, (11) flow through a rectangular duct when the conducting part is symmetrically situated, and (11i) flow through a rectangular duct when the conducting part is arbitrarily positioned.

Such problems have been studied before by asymptotic means for large values of $M$, the Hartmann number. However, the present modification of the Boundary Integral Method renders the problem computationally efficient and provides a reliable numerical solution for all values of $M$. For large $M$, our computation $t$ ime decreases significantly.

KEY WORDS AND NEW PHRASES. Boundary integral method, MHD flow, and asymptotic solutions.

1980 AMS SUBJECT CLASSIFICATION CODE. 76 W05.

\section{INTRODUCTION.}

The MHD flow of an electrically conducting fluid through ducts in the presence of a magnetic field is of impotance in varlous areas of technology and engineering such as MHD power generation, MHD flow-meters, MHD pumps etc. Since the classical works by Hartmann [1] and Shercliff [2], many Investigations have been carried out in which various combinations of boundary conditions have been considered [3-14]. Special attention has been paid in these investigations to large values of $M$, the Hartmann number, to study the formation of varlous boundary layers in the field at large M. 
Hunt and Willians [15] studied the effects of a discontinuity in the electromagnetic boundary conditions by considering the flow driven by the currents produced by electrodes placed in each plane of flat channel. The authors [16, 17] recently extended this idea and investigated the MHD flow through ducts in which one of the boundaries perpendicular to the magnetic field had mixed electromagnetic boundary conditions; by reducing the problem to the solution of a Fredholm Integral equation.

In the present paper, we investigate the MHD flow through ducts, when one of the boundaries perpendicular to the magnetic field has a portion of the boundary perfectly conducting and the remaining boundaries are insulated, by the Boundary Integral Method. Three geometries are considered (1) an infinite channe1, (11) a rectagnular duct when the conducting part is symmetrically situated, and (iii) a rectangular duct when the conducting part is arbitrarily positioned. The usual boundary integral method has been modified to develop an integral equation for the value of the magnetic field on the conducting part of the boundary, which integral equation has a singularity no stronger than $\ln \mathrm{r}$. This integral equation has been solved numerically and the results presented for various values of the Hartmann number. Such problems would arise, e.g., in coupled MHD ejectors.

\section{THE EQUATIONS OF MOTION.}

Consider the flow of a viscous, incompressible, electrically conducting fluid through a duct. The flow is driven by a constant pressure gradient and is assumed to be fully developed, steady and laminar. A uniform magnetic field is applied perpendicular to the boundary with mixed electromagnetic boundary conditions.

The equation of motion and the curl of Ohm's law in the dimensionless form are (Shercliff [2], Dragos [18])

$$
\begin{aligned}
& \nabla^{2} V+M \frac{\partial B}{\partial x}=-1 \\
& \nabla^{2} B+M \frac{\partial V}{\partial x}=0
\end{aligned}
$$

Here $V$ is the velocity in the $z$-direction, $B$ is the induced magnetic field and $M$ is the Hartmann number defined by

$$
M=\mu_{e} H_{0} L_{\star}(\sigma / \mu)^{1 / 2}
$$

where $L_{\star}$ is a characteristic length and $\mu, \sigma$ and $\mu_{e}$ respectively denote the coefficient of viscosity, electrical conductivity and magnetic permeability. $H_{0}$ is the strength of the applied magnetic field directed along $x$-axis.

3. SOLUTION FOR PRIMARY FLOW.

Taking the primary flow as corresponding to perfectly insulated walls, its solution is given by

$$
v_{0}(x)=\frac{a}{2 M \operatorname{sh} \frac{1}{2} M a}\left[\operatorname{ch} \frac{1}{2} M a-\operatorname{ch} M\left(\frac{a}{2}-x\right)\right]
$$




$$
B_{0}(x)=\frac{1}{M}\left(\frac{a}{2}-x\right)-\frac{a \operatorname{sh} M\left(\frac{1}{2} a-x\right)}{\operatorname{sh~} \frac{1}{2} M a}
$$

for an infinite channel bounded by the planes $x=0$ and $x=a$.

The solution for a rectangular duct bounded by the planes $x=0, x=a$ and $y= \pm$ b is

and

$$
v_{0}(x, y)=\sum_{m=1,3}^{\infty} v_{m}(x) \sin \frac{m \pi}{2} \cos \frac{m \pi y}{2 b}
$$

$$
B_{0}(x, y)=\sum_{m=1,3}^{\infty} b_{m}(x) \sin \frac{m \pi}{2} \cos \frac{m \pi y}{2 b}
$$

where

and

$$
\begin{aligned}
& v_{m}(x)=\frac{16 b^{2}}{m^{3} \pi^{3}}\left[1-\frac{s h \rho_{m} x \operatorname{ch} \frac{1}{2} M(a-x)+s h \rho_{m}(a-x) c h \frac{1}{2} M x}{s h \rho_{m} a}\right] \\
& b_{m}(x)=\frac{16 b^{2}}{m^{3} \pi^{3}} \cdot \frac{s h \rho_{m}(a-x) \operatorname{sh} \frac{1}{2} M x-s h \rho_{m} x \operatorname{sh} \frac{1}{2} M(a-x)}{s h \rho_{m}^{a}}
\end{aligned}
$$

$$
\rho_{m}^{2}=\frac{m^{2} \pi^{2}}{4 b^{2}}+\frac{M^{2}}{4}
$$

For a rectangular duct bounded by the planes $x=0, x=a, y=0$ and $y=b$, the primary solution is easily obtained from equations (3.3) and (3.4) by a suitable change of variable.

We shall examine the effect of the perfectly conducting part of the boundary on the flow in the following sections. Thus we write the solution for the flow as

$$
\left\{\begin{array}{l}
v \\
B
\end{array}\right\}=\left\{\begin{array}{l}
v_{0} \\
B_{0}
\end{array}\right\}+\left\{\begin{array}{c}
v_{1} \\
B_{1}
\end{array}\right\} \text {. }
$$

where $V_{1}$ and $B_{1}$ are the velocity and magnetic field induced by the electrical conductivity of the boundary.

4. SOLUTION FOR SECONDARY FLOW.

The equations of motion for secondary flow and the associated boundary conditions are

$$
\begin{aligned}
& \nabla^{2} v_{1}+M \frac{\partial B_{1}}{\partial x}=0 \\
& \nabla^{2} B_{1}+M \frac{\partial v_{1}}{\partial x}=0 \\
& v_{1}=0 \quad \text { on the boundaries } \\
& B_{1}=0 \quad \text { on the non-conducting boundaries } \\
& \frac{\partial B_{1}}{\partial n}=-\frac{\partial B_{0}}{\partial n} \quad \text { on the conducting part of the mixed boundary }
\end{aligned}
$$


where $\partial / \partial n$ denotes the derivative in the normal direction.

A solution of equations $(4.1)$ and $(4.2)$ is

and

$$
v_{1}(x, y)=-\operatorname{sh} \frac{M x}{2} f(x, y)
$$

$$
B_{1}(x, y)=\operatorname{ch} \frac{M x}{2} f(x, y)
$$

where the function $f(x, y)$ satisfies the differential equation

$$
\nabla^{2} \mathrm{f}-\frac{1}{4} \mathrm{M}^{2} \mathrm{f}=0
$$

and the boundary conditions

$$
\begin{aligned}
& f=0 \text { on the non-conducting boundaries } \\
& \frac{\partial f}{\partial x}(0, y)=-\frac{\partial B}{\partial x}(0, y) \quad \text { on the conducting part. }
\end{aligned}
$$

The appropriate solution of equation (4.6) in terms of the Green's functions $G$ is given by

$$
f(P)=\frac{1}{2 \pi} \int\left\{f(Q) \frac{\partial}{\partial n_{Q}} G(P, Q)-G(P, Q) \frac{\partial f}{\partial n_{Q}}\right\} d s_{Q}
$$

Here $P$ is any point interior to the domain $D, Q$ is any point on the boundary $\partial D$ of the domain $D$ and $\partial / \partial n_{Q}$ denotes the derivative in the direction of the outward drawn normal. $G$ is a suitable Green's function.

\subsection{THE FLOW IN AN INFINITE CHANNEL.}

Assume that the conducting part of the boundary $x=0$ is symmetrically situated at $|y|<c_{\text {. }}$ Because of the symmetry of the problem, we need to consider the solution in the region $y>0$ only. The boundary condition at $y=0$ becomes

$$
\frac{\partial f}{\partial y}(x, 0)=0
$$

Choosing

$$
\begin{aligned}
& G(x, y ; \xi, n)=\sum_{m=-\infty}^{\infty}(-1)^{m}\left\{k_{0}\left[\frac{M}{2} \sqrt{\left(x_{m}-\xi\right)^{2}+(y-n)^{2}}\right]+\right. \\
& \left.+K_{0}\left[\frac{M}{2} \sqrt{\left(x_{m}-\xi\right)^{2}+(y+n)^{2}}\right]\right\}
\end{aligned}
$$

where

$$
x_{m}=\left(m+\frac{1}{2}\right) a+(-1)^{m}\left(x-\frac{1}{2} a\right)
$$

equations (4.9), (4.4) and (4.5) give 
and

$$
B_{1}(x, y)=\frac{1}{2 \pi} \operatorname{ch} \frac{M x}{2} \int_{0}^{c} B_{1}(0, n) \frac{\partial G}{\partial \xi}(x, y ; 0, n) d n
$$

$$
v_{1}(x, y)=-\frac{1}{2 \pi} \operatorname{sh} \frac{M x}{2} \int_{0}^{c} B_{1}(0, n) \frac{\partial G}{\partial \xi}(x, y: 0, n) d n
$$

Notice that $\frac{\partial G}{\partial \xi}$ has a singularity of the type $\frac{1}{r}$ at $x=0, y=\eta$, and we still need to differentiate it.

\subsection{MODIFIED INTEGRAL EQUATION FOR B . $_{1}$}

Differentiating equation (4.13) with respect to $x$ and taking limits as $x$ tends to zero, we obtain from $(4.3 \mathrm{c})$

$$
\lim _{x \rightarrow 0} \int_{0}^{c} B_{1}(0, n) \frac{\partial^{2} \bar{G}}{\partial \xi^{2}}(x, y ; 0, n) d n=2 \pi \frac{\partial B}{\partial x}(0, y)
$$

where $\bar{G}$ is given by

$$
\begin{aligned}
& \bar{G}(x, y ; \xi, n)=\sum_{m=-\infty}^{\infty}\left\{k_{o}\left[\frac{M}{2} \sqrt{\left(x_{m}-\xi\right)^{2}+(y-n)^{2}}\right]+\right. \\
& \left.+K_{0}\left[\frac{M}{2} \sqrt{\left(x_{m}-\xi\right)^{2}+(y+n)^{2}}\right]\right\}
\end{aligned}
$$

Since the function $\bar{G}$ also satisfies the differential equation (4.6), equation (4.15) can be rewritten as

$$
\lim _{x \rightarrow 0} \int_{0}^{c} B_{1}(0, n)\left[\frac{1}{4} M^{2} G(x, y ; 0, n)-\frac{\partial^{2} \bar{G}}{\partial \eta^{2}}(x, y ; 0, n)\right] d n=2 \pi \frac{\partial B}{\partial x}(0, y)
$$

Integrating the second term in the integrand by parts and noting that $B_{1}$ vanishes at $n=c$ and $\frac{\partial \bar{G}}{\partial \eta}$ vanishes at $n=0$, equation $(4.17)$ reduces to

$$
\lim _{x \rightarrow 0} \int_{0}^{c}\left[\frac{1}{4} M^{2} B_{1}(0, n) \bar{G}(x, y ; 0, n)+B_{1}^{\prime}(0, n) \frac{\partial \bar{G}}{\partial \eta}(x, y ; 0, n)\right] d n=2 \pi \frac{\partial B}{\partial x}(0, y)
$$

where a prime(') denotes the derivative with respect to $n$.

Ass ume

$$
g(n)=\int_{0}^{n} B_{1}(0, y) d y
$$

which implies

$$
g(0)=0
$$

and

$$
g^{\prime}(n)=B_{1}(0, n)
$$

Equation ( 4.18 ) now leads to

$$
\lim _{x \rightarrow 0} \int_{0}^{c}\left[\frac{1}{4} M^{2} g^{\prime}(n) \bar{G}(x, y ; 0, n)+g^{\prime \prime}(n) \frac{\partial \bar{G}}{\partial \eta}(x, y ; 0, n)\right] d n=2 \pi \frac{\partial B}{\partial x}(0, y)
$$


Integrating first term in the integrand by parts and using (4.20) we have

$$
\lim _{x \rightarrow 0}\left[\frac{1}{4} M^{2} g(c) \bar{G}(x, y ; 0, c)-\int_{0}^{c} h(n) \frac{\partial G}{\partial y}(x, y ; 0, n) d n\right]=2 \pi \frac{\partial B}{\partial x}(0, y)
$$

where the function $G$ is given by

$$
\begin{aligned}
& G(x, y ; \xi, n)=\sum_{\mathbb{m}=-\infty}^{\infty}\left\{K_{0}\left[\frac{M}{2} \sqrt{\left(x_{m}-\xi\right)^{2}+(y-n)^{2}}\right]-\right. \\
& \left.-K_{0}\left[\frac{M}{2} \sqrt{\left(x_{m}-\xi\right)^{2}+(y+n)^{2}}\right]\right\}
\end{aligned}
$$

and

$$
h(n)=g^{\prime \prime}(n)-\frac{1}{4} M^{2} g(n)
$$

Integrating equation (4.23) with respect to $\mathrm{y}$ from 0 to $\mathrm{y}$ and taking the limits, we finally obtain the following integral equation for $h$

$$
\int_{0}^{c} h(n) G(0, y ; 0, v) d n=g(c) P(y)+Q(y)
$$

where

and

$$
P(y)=\frac{M^{2}}{4} \int_{0}^{y} \bar{G}\left(0, n ; 0, c_{2}\right) d_{n}
$$

$$
Q(y)=2 \pi \int_{0}^{y} \frac{\partial B}{\partial x}(0, n) d n
$$

Equation (4.26) is the integral equation we need to solve. One should note that this equation has a singularity of the type $\ln r$ which can be handled by the usual boundary integral technique. Also, equation (4.26) needs to be solved on $1 y$ on the conducting part of the boundary, and not over the whole boundary, and this leads to significant saving in computation. However, the constant $g(c)$ still needs to be determined. $g(c)$ is calculated as follows:

Let

$$
h(n)=g(c) h_{1}(n)+h_{2}(n)
$$

where $h_{1}$ and $h_{2}$ satisfy

$$
\begin{aligned}
& \int_{0}^{c} h_{1}(n) G(0, y: 0, n) d n=P(y) \\
& \int_{0}^{c} h_{2}(n) G(0, y ; 0, n) d n=Q(y)
\end{aligned}
$$

respective1y.

Also let

$$
g(n)=g(c) g_{1}(n)+g_{2}(n)
$$

where $g_{i}(n)$ satisfy the BVP 


$$
\begin{gathered}
g_{i} \prime(n)-\frac{1}{4} M^{2} g_{i}(n)=h_{i}(n) \\
\text { with } g_{i}(0)=0, g_{i}^{\prime}(c)=0 \quad(i=1,2)
\end{gathered}
$$

By solving equations (4.30) and (4.31) for $h_{i}(n)$, one can obtain $g_{i}(n)$ from equation (4.33). Now $g(c)$ can be obtained from equation (4.32) by using the condition $g(c)=g(c)$. We have

$$
g(c)=\frac{g_{2}(c)}{1-g_{1}(c)}
$$

Having obtained $g(c)$ and $g_{1}(n), B_{1}(0, n)$ can be calculated from equations (4.32) and $(4.21)$.

\subsection{NUMERICAL COMPUTATIONS.}

Equations (4.30) and (4.31) were solved numerically by descretizing the interval $(0, c)$ into a mesh and using an analog of Euler's mid-point rule for integration

$$
\int_{n_{j}}^{n} j+1 h(n) G(0, y ; 0, n) d n=h\left(n_{j+1 / 2}\right) \int_{n_{j}}^{n} G(0, y ; 0, n) d n
$$

Thus the values of $h$ were calculated at the mid-points of the mesh-points rather than at the nodal points. The values of $y$ were also chosen at these same points. The resulting linear algebraic system of equations was solved on the Cyber 860 at the University of Calgary, Calgary, Canada, invoking the IMSL subroutine LEQTIF which uses the Gauss elimination method with partial pivoting.

Note that $G$ has a singular part of the form $K_{0}\left(\frac{1}{2} M r\right)$ when $y=n$. This part was first integrated by parts resulting into an integrand without a singularity but which varies sharpiy for large $M$, near $r=0$. This was, therefore, computed accurately using the IMSL routine DCADRE. The remaining integrals, being free of singularities, were evaluated using the three point Gauss Legendre formula, which was sufficient to produce desired accuracy.

The BVP (4.33) was solved using Green's functions (rather than the shooting method which would cause difficulties for large $M$ ). Once again an analog of Euler's mid-point formula was used to calculate the integrals and this enabled us to compute the values of $B_{1}(0, n)$ at the nodal points of the mesh.

The values of $B_{1}(0, n)$ were calculated using 50 and 100 mesh-points. To further improve the accuracy of the results, Richardson's extrapolation was used. The improved values of $B_{1}(0, n)$ were used to compute the secondary $f 1$ ow given by equations (4.13) and (4.14), using the subroutine DCADRE, once again.

\subsection{RESULTS AND DISCUSSION.}

The velocity distribution and magnetic field were calculated for $c=0.2$ and various values of $M$. This problem has been investigated by the authors [17] recently using the Fourier transform technique, and the present results for these cases are in good agreement with the results obtained there. However some difficulties were 
encountered there for large values of $M$. Such difficulties were not encountered here. Also worth mentioning is the fact that using the present method the execution time reduced with increasing values of $M$ as fewer terms are needed for computation of the expression for the Green's function G. For $M=10$, it took 1.950 seconds of CPU time on he Cyber 860 for calculating $B_{1}(0, \eta)$ for a single run, but for $M=50$ it took 0.485 seconds, and 0.315 seconds for $M=100$. Indeed this time could have been further reduced by using the Gauss-Seidel method instead of Gauss elimination, as for large values of $M$ the algebraic system of equations determining $h$ is highly diagonally dominant.

In Figure 2, velocity contours are presented for $M=100$. As expected, boundary layer formation takes place for large values of $M$ near the non-conducting boundaries perpendicular to the magnetic field. Also shear layers [16, 17, 19] given by

$$
|y \pm c|=\frac{2}{\sqrt{M}} \sqrt{x}
$$

which emanate from the points of discontinuity in the electromagnetic boundary conditions are evident in the figure. This shear layer has been depicted in Figure 2 by means of dashed 11 nes.

In Figure 3, the current lines (constant magnetic field 1ines) are presented for $M=100$. Again, one can notice the formation of the two boundary layers for large values of $M$. The parabolic shear layer is demarcated by dashed lines. It may be noted that the changes in the values of magnetic field take place mostly in this parabolic layer.

4.5. THE FLOW IN A RECTANGULAR DUCT SYMMETRIC CASE.

Again, we assume that the conducting part of the boundary $x=0$ is symetrically placed at $|y|<c$. The only difference in the analysis from the preceeding case is in the expressions for Green's functions. They are now defined as

$$
\begin{aligned}
& G(x, y ; \xi, n)=\sum_{\mathbb{m}=-\infty}^{\infty} \sum_{n=-\infty}^{\infty}(-1)^{m+n^{\prime}} k_{0}\left[\frac{M}{2} \overline{\sqrt{\left(x_{m}-\xi\right)^{2}+\left(y_{n}-n\right)^{2}}}\right] \\
& \bar{G}(x, y ; \xi, n)=\sum_{m=-\infty}^{\infty} \sum_{n=-\infty}^{\infty}(-1)^{n^{\prime}} k_{0}\left[\frac{M}{2} \sqrt{\left(x_{m}-\xi\right)^{2}+\left(y_{n}-\eta\right)^{2}}\right] \\
& G(x, y ; \xi, n)=\sum_{m=-\infty}^{\infty} \sum_{n=-\infty}^{\infty}(-1)^{n "} k_{0}\left[\frac{M}{2} \sqrt{\left(x_{m}-\xi\right)^{2}+\left(y_{n}-n\right)^{2}}\right]
\end{aligned}
$$

where

and

$$
y_{n}=\left(n+\frac{1}{2}\right) b+(-1)^{n}\left(y-\frac{1}{2} b\right)
$$

$$
n^{\prime}=\left(\frac{n+1}{2}\right), n^{\prime \prime}=\left(\frac{n}{2}\right)
$$

[x] being the integral part of $x$.

In Figure 4 and 5, equal velocity lines and current lines are presented respectively, for $c=0.2$ and $M=100$. The parabolic shear layers gien by equation (4.36) have been demarcated by dashed lines. 
4.6. THE FLOW IN A RECTANGULAR DUCT NON-SYMMETRIC CASE.

In this case the positioning of the conducting part is taken arbitrary i.e., $c_{1}<y<c_{2}$. The analysis now is slightly different compared with the previous cases.

The expressions for velocity and magnetic field are

$V_{1}(x, y)=-\frac{1}{2 \pi} \operatorname{sh} \frac{M x}{2} \int_{c_{1}}^{c_{2}} B_{1}(0, n) \frac{\partial G}{\partial \xi}(x, y ; 0, n) d \eta$

$$
B_{1}(x, y)=\frac{1}{2 \pi} \operatorname{ch} \frac{M x}{2} \int_{c_{1}}^{c_{2}} B_{1}(0, n) \frac{\partial G}{\partial \xi}(x, y ; 0, n) d n
$$

where

$$
G(x, y ; \xi, n)=\sum_{m=-\infty}^{\infty} \sum_{n=-\infty}^{\infty}(-1)^{m+n} K_{0}\left[\frac{M}{2} \sqrt{\left(x_{m}-\xi\right)^{2}+\left(y_{n}-n\right)^{2}}\right]
$$

$x_{m}$ and $y_{n}$ being defined by equations (4.12) and (4.40) respectively.

$$
\text { Defining } g(n)=\int_{c_{1}}^{n} B_{1}(0, y) d y
$$

and proceeding as in Section 4.2, we obtain the following integral equation for $h(\eta)$ $\begin{array}{ll}\text { where } & \int_{c_{1}}^{c_{2}} h(n) G(0, y ; 0, n) d n=g\left(c_{2}\right) P(y)+Q(y)+A \\ & P(y)=\frac{M^{2}}{4} \int_{c_{1}}^{y} \bar{G}\left(0, n ; 0, c_{2}\right) d n \\ \text { and } & Q(y)=-2 \pi \int_{c_{1}}^{\frac{\partial B}{O}} \frac{{ }_{0}}{\partial x}(0, n) d n\end{array}$

$$
A=\int_{c_{1}}^{c_{2}} h(\eta) G\left(0, c_{1} ; 0, n\right) d n
$$

The functions $\bar{G}$ and $G$ now become

and

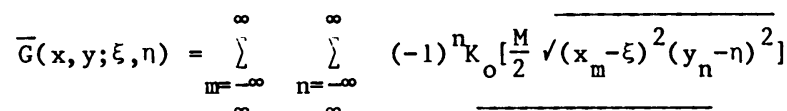

$$
\begin{aligned}
& G(x, y ; \xi, n)=\sum_{m=-\infty}^{\infty} \sum_{n=-\infty}^{\infty} k_{0}\left[\frac{M}{2} \sqrt{\left(x_{m}-\xi\right)^{2}+\left(y_{n}-n\right)^{2}}\right]
\end{aligned}
$$

Now there are two constants $A$ and $g\left(c_{2}\right)$ which need to be calculated. Let

$$
h(n)=g\left(c_{2}\right) h_{1}(n)+h_{2}(n)+A h_{3}(n)
$$

where $h_{1}, h_{2}$ and $h_{3}$ are given by

$$
\int_{c_{1}}^{c} h_{1}(n) G(0, y ; 0, n) d n=P(y)
$$




$$
\begin{aligned}
& \int_{c_{1}}^{c_{2}} h_{2}(n) G(0, y ; 0, n) d n=Q(y) \\
& \int_{c_{1}}^{c_{1}} h_{3}(n) G(0, y ; 0, n) d n=1
\end{aligned}
$$

respectively.

Further assume

$$
g(n)=g\left(c_{2}\right) g_{1}(n)+g_{2}(n)+A_{3}(n)
$$

where $g_{1}(n)$ satisfy the BVP

$$
g_{1}^{\prime \prime}(n)-\frac{1}{4} M^{2} g_{i}(n)=h_{1}(n)
$$

with

$$
g_{i}^{\prime}\left(c_{1}\right)=0, g_{i}^{\prime}\left(c_{2}\right)=0(1=1,2,3)
$$

The constants $A$ and $g\left(c_{2}\right)$ can now be obtained by using the condition $g\left(c_{1}\right)=0$ and the trivial condition $g\left(c_{2}\right)=g\left(c_{2}\right)$. We have

and

$$
A=\frac{g_{1}\left(c_{1}\right) g_{2}\left(c_{2}\right)-g_{2}\left(c_{1}\right)\left[g_{1}\left(c_{2}\right)-1\right]}{g_{3}\left(c_{1}\right)\left[g_{1}\left(c_{2}\right)-1\right]-g_{3}\left(c_{2}\right) g_{1}\left(c_{1}\right)}
$$

$$
g\left(c_{2}\right)=\frac{g_{2}\left(c_{1}\right) g_{3}\left(c_{2}\right)-g_{3}\left(c_{1}\right) g_{2}\left(c_{2}\right)}{g_{3}\left(c_{1}\right)\left[g_{1}\left(c_{2}\right)-1\right]-g_{3}\left(c_{2}\right) g_{1}\left(c_{1}\right)}
$$

In Figures 6 and 7 , equal velocity lines and current lines are shown respectively, for $c_{1}=0, c_{2}=0.2$ and $M=100$. The shear layer has been indicated by dashed 1ines. One should note the interaction of various layers in these figures. Such interaction would be difficult to obtain by asympotic methods.

Finally in Figure 8 , the values of $B(0, y)$ are plotted on the conducting part for the geometries considered in this paper.

\section{ACXNOWL EDGEMENTS}

Financial support of the Natural Sciences and Engineering Research Council of Canada is gratefully acknowledged. 


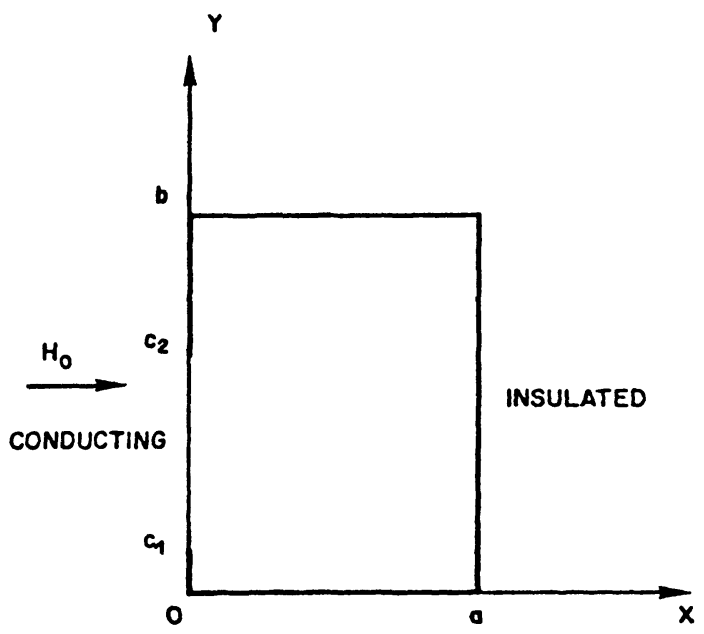

Figure 1. Geometry of the duct.

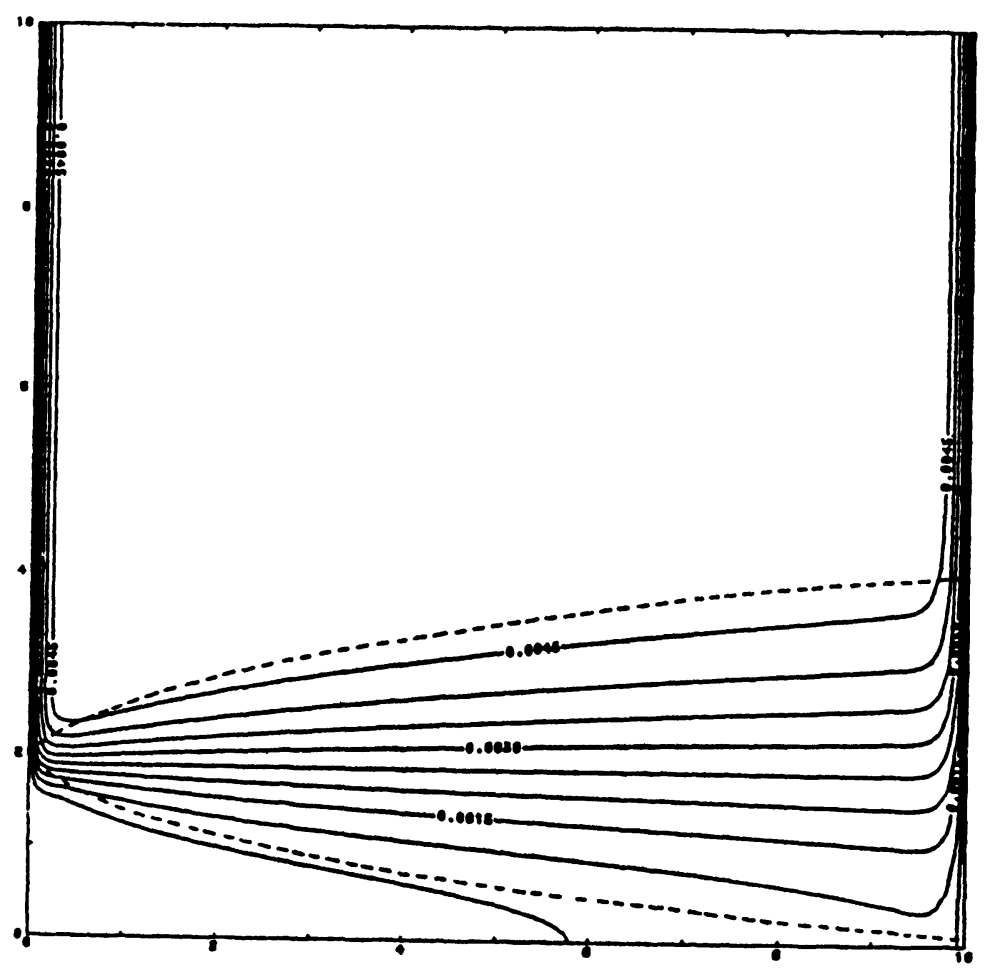

Figure 2. Equal velocity lines in infinite channel $(y \geq 0)$ for $M=100, a=1, c=0.2$. The parabolic boundary layer is indicated by dashed lines. 


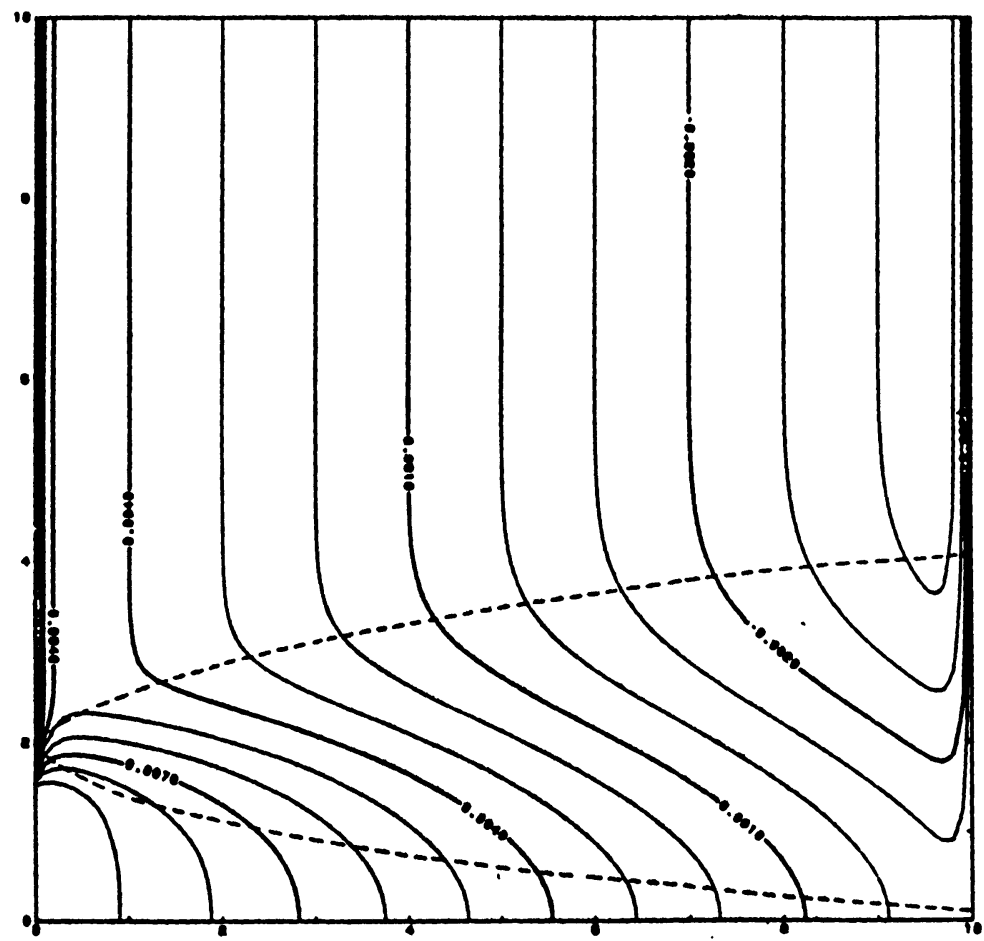

Figure 3. Equal magnetic field lines in infinite channel $(y \geq 0)$ for $M=100, a=1, c=0.2$. The parabolic boundary layer is indicated by dashed lines.

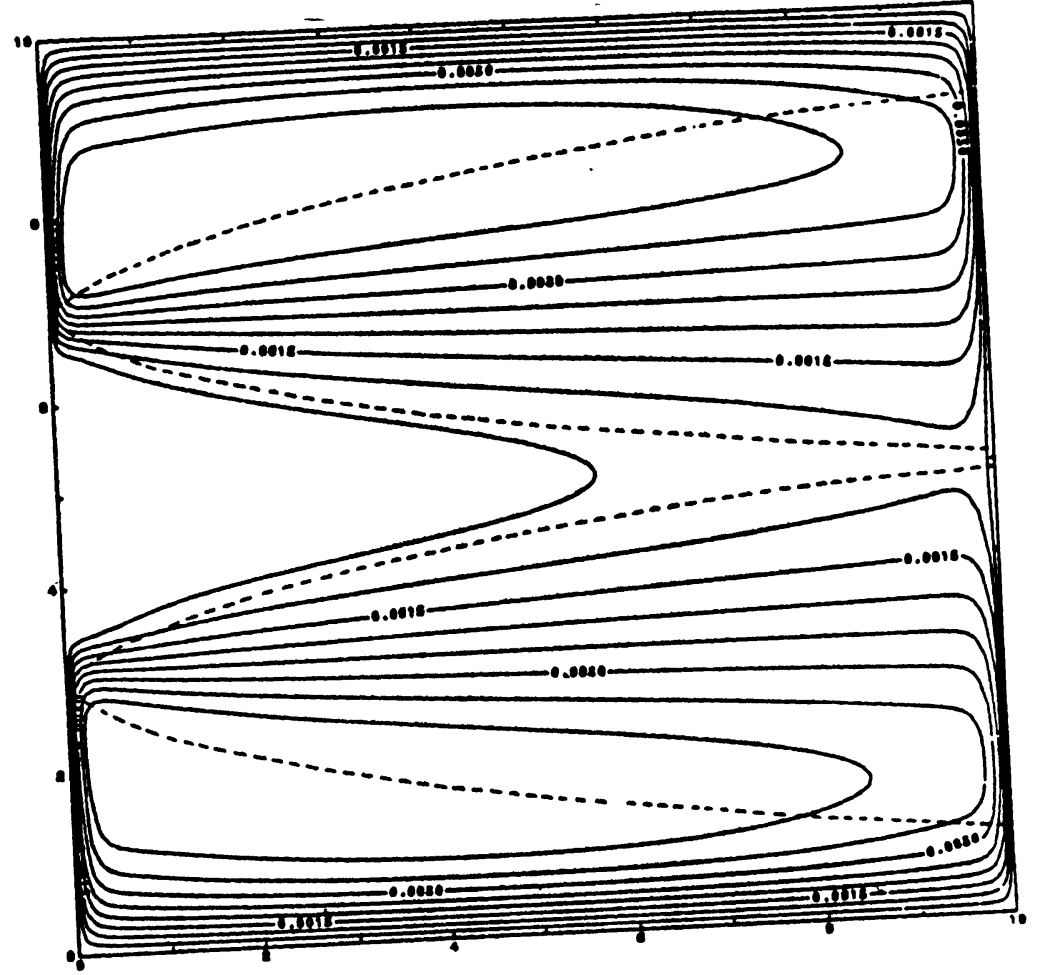

Figure 4. Equal velocity lines in the rectangular duct (symetric case) for $M=100, a=b=1, c=0.2$. The parabolic boundary layer is indicated by dashed ines. 


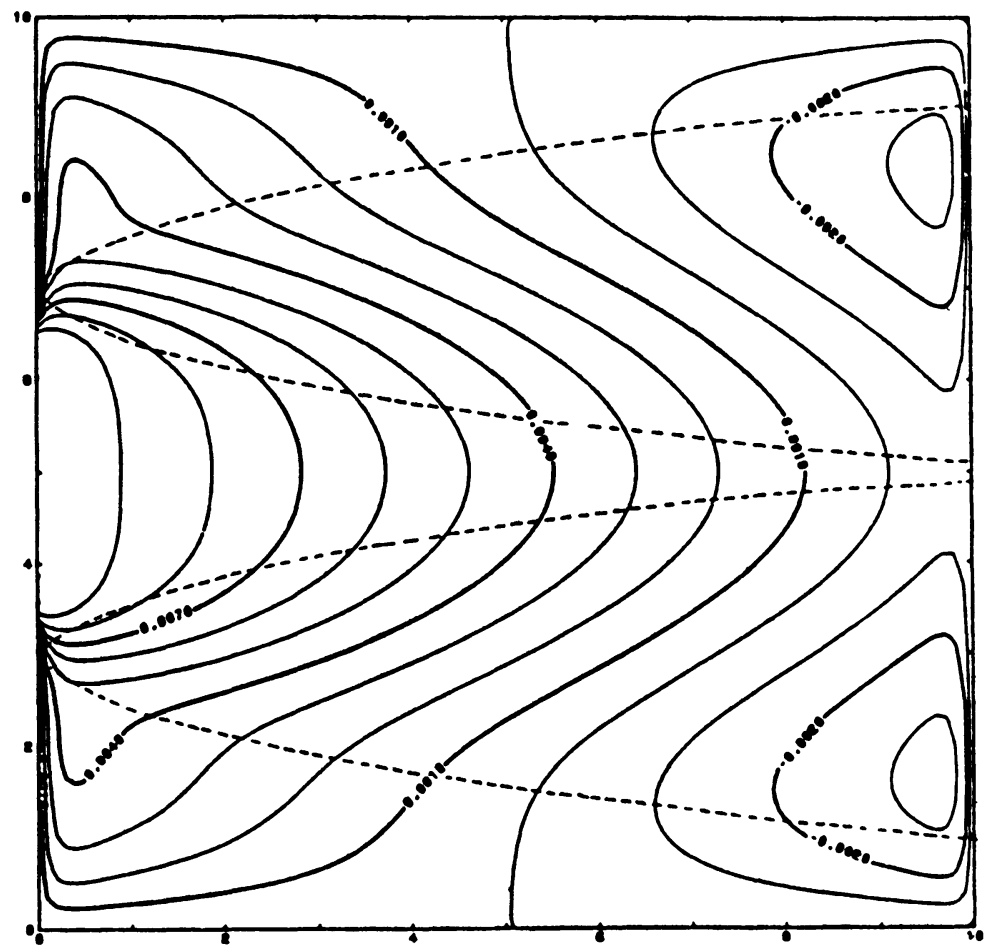

Figure 5. Equal magnetic field lines in the rectangular duct (symmetric case) for $M=100, a=b=1, c=0.2$.

The parabolic boundary layer is indicated by dashed lines.

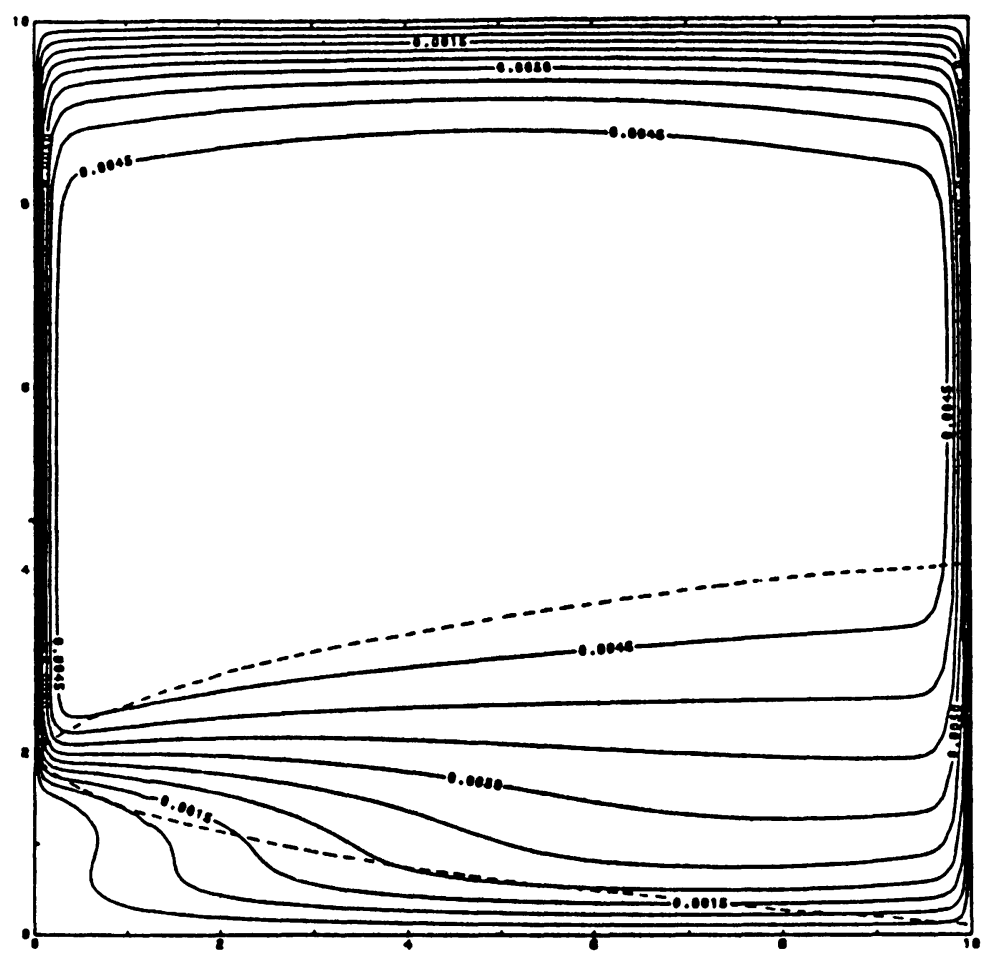

Figure 6. Equal velocity lines in the rectangular duct (non-symmetric case) for $M=100, a=b=1$, $c_{1}=0, c_{2}=0.2$. The parabolic boundary layer is indicated by dashed lines. 


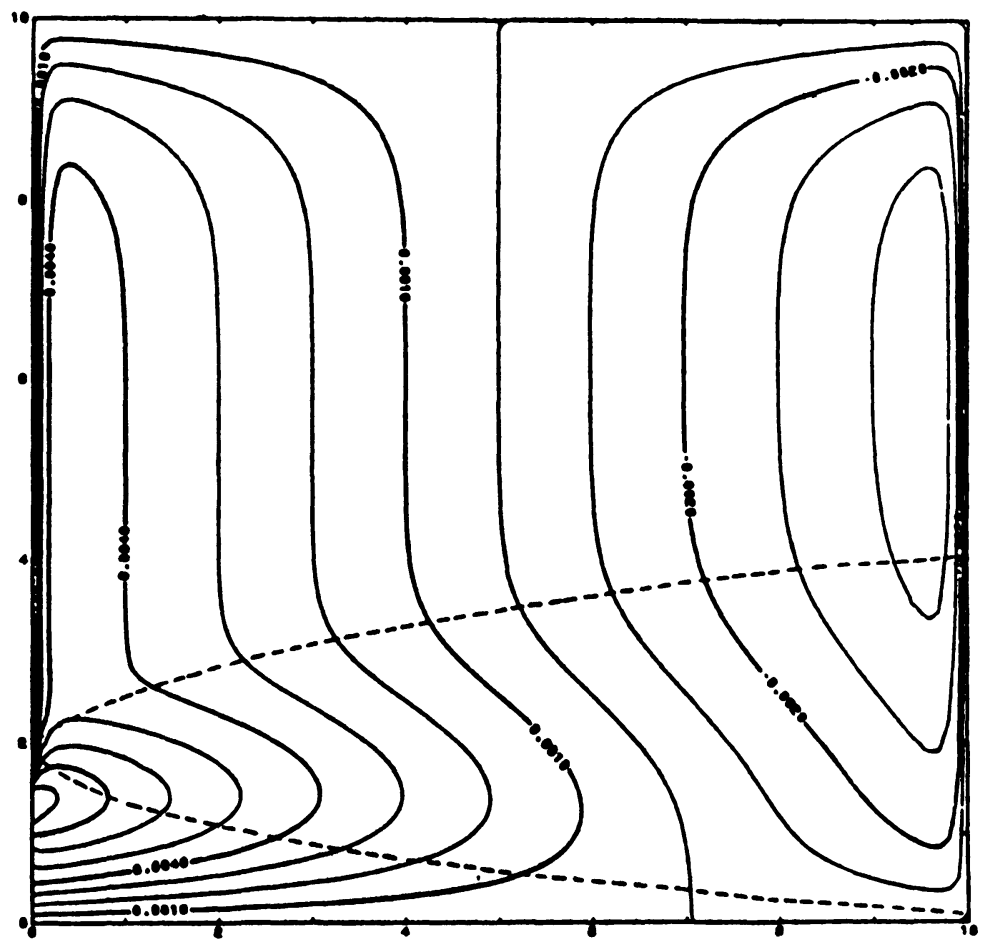

Figure 7. Equal magnetic field lines in the rectangular duct (non-syumetric case) for $M=100, a=b=1$, $c_{1}=0, c_{2}=0.2$. The parabolic boundary layer is indicated by dashed lines. 


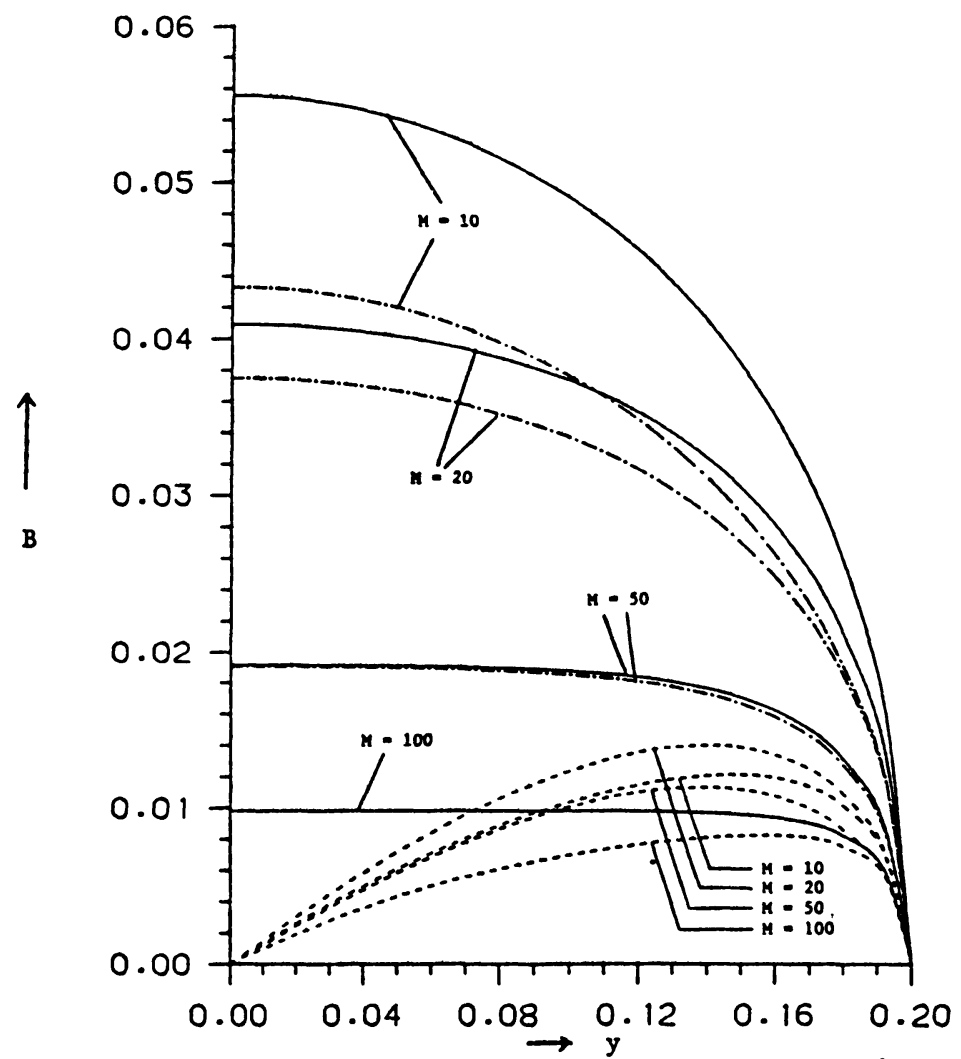

Figure 8. Values of $B$ on the conducting part of the boundary $x=0$ for various values of $M$. The curves represent $B$ for infinite channel, the curves -.-.-. represent $B$ for rectansular duct (symmetric-case) and the curves - - - represent $B$ for rectangular duct (non-symmetric case). 


\section{REFERENCES}

1. HARTMANN, J.,Hg-Dynamics-I, Theory of the Laminar Flow of an Electrically Conducting Liquid in a homogeneous Magnetic Field, Math. Fys. Medd.,15 6 (1937).

2. SHERCLIFF, I. Steady Motion of Conducting Fluids in Pipes under Transverse Magnetic Fields, Proc. Camb. Phil. Soc. 49, (1953), 136-144.

3. HUNT, J.C.R., Magnetohydrodynamic Flow in Rectangular Ducts, Jour. Flu1d Mech. 21 (1965), 577-590.

4. GRINBERG, G.A., On Steady Flow of a Conducting Fluid in a Rectangular Tube with two Nonconducting Walls, and two Conducting Ones Parallel to an External Magnetic Field, PMM, 25 (1961), 1024-1034.

5. GRINBERG, G.A., On Some Types of Flow of a Conducting Fluid in P1pes of Rectangular Cross section, Placed in a Magnetic Field PMM, 26 (1962), 80-87.

6. HUNT, J.C.R. and STEWARTSON, K., Magnetohydrodynamic Flow in Rectangular Ducts II Jour. Fluid Mech. 23, (1965), 563-581.

7. CHIANG, D. and LUNDGREN, T., Magnetohydrodynamic Flow in a Rectangular Duct with Perfectly Conducting Electrodes, ZAMP, 18, (1967), 92-105.

8. LAVRENTEV, I.V. and SHISKO, A. YA., Electrodynamic Processes in MHD Channels with High Magnetic Reynold's Number, MHD, 16 (1980), 88-311.

9. SINGH, BANI and AGARWAL, P.K., Numerical Solution of a Singular Integral Equation Appearing in Magnetohydrodynamics, ZAMP, 35 (1984), 760-770.

10. SINGH, BANI and LAL JIA, Kantorovich Method in Magnetohydrodynamic Flow Problems through Channe18, Ind. Jour. Pure App1. Math., 15, (1984), 10481063.

11. AITOV, T.N., KALYUTIK, A.I. and TANANAEV, A.V., Numerical St udy of ThreeDimensional MHD Flow in a Duct of Complex Configuration, Using the Stokes Approximation, MHD, 20, (1984), 288-293.

12. KASHEVSKII, B.E. and NOGOTOV, E.F., Non-Linear MHD Effects Found in the Channel Flow of a Magnetic Liquid, MHD, 20 (1984), 339-342.

13. ANTIMIROV, M. YA., Solution of MHD Problems with Mixed Boundary Conditions, MHD 21 (1985), 116-121.

14. SLATINSKI, N.D., SMIRNOV, V.I., FEDONENKO, A.I. and TARAPOV, I.E., F1 Ow of Electrically Conducting Magnetic Liquid with Generalized Rheological Law in a Plane Channe1, MHD, 22 (1986), 155-161.

15. HUNT, J.C.R. and WILLIAMS, W.E., Some Electrically Driven Flows in Magnetohyd rodynamics, Part I., Theory, J. Fluid Mech., 31, (1968), 705-722.

16. SEZGIN, M., AGGARWALA, B.D. and ARIEL, P.D., Electrically Driven Flows in MHD With Mixed Electromagnetic Boundary Conditions, ZAMM, (1987), to appear.

17. SEZGIN, M., ARIEL, P.D. and AGGARWALA, B.D., Pressure Driven MHD Flows in Channels with One Boundary Partially Conducting and Partially Insulating (Submitted for Publication).

18. DRAGOS, L., Magnetofluid Dynamics, Abacus Press, 1975.

19. HUNT, J.C.R. and SHERCLIFF, J.A., Magnetohyd rodynamics at High Hartmann number Annua1 Rev. Fluid Mech. 3 (1971), 37-62. 


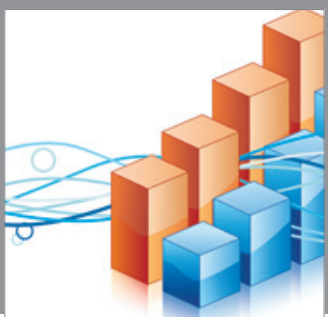

Advances in

Operations Research

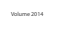

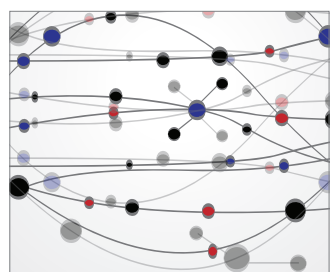

\section{The Scientific} World Journal
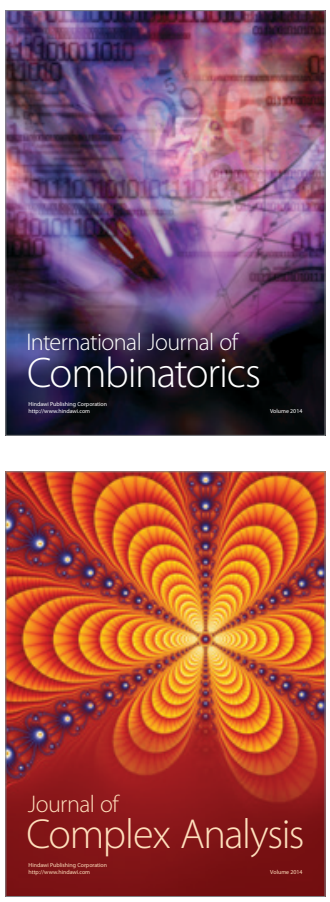

International Journal of

Mathematics and

Mathematical

Sciences
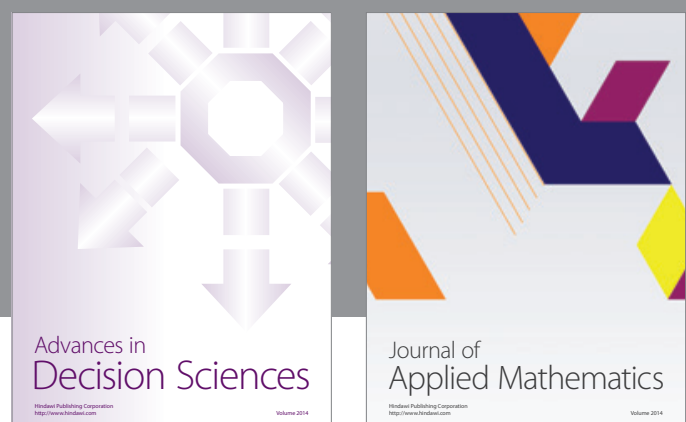

Journal of

Applied Mathematics
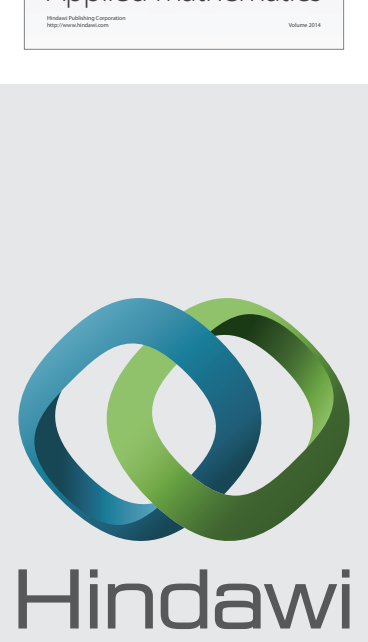

Submit your manuscripts at http://www.hindawi.com
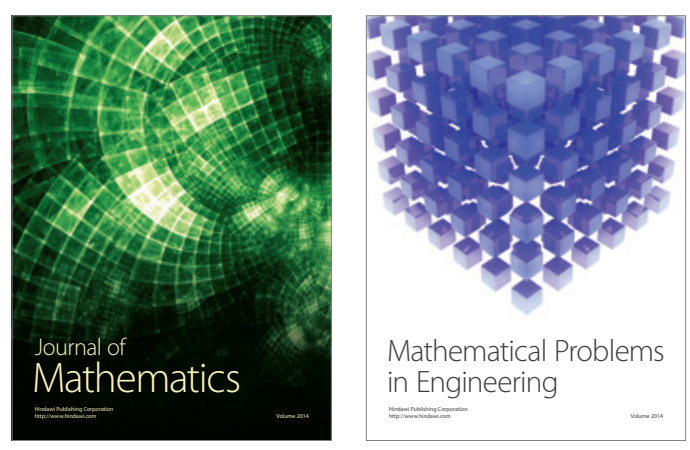

Mathematical Problems in Engineering
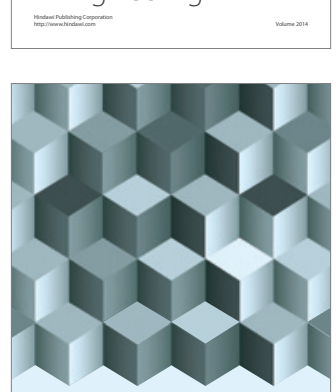

Journal of

Function Spaces
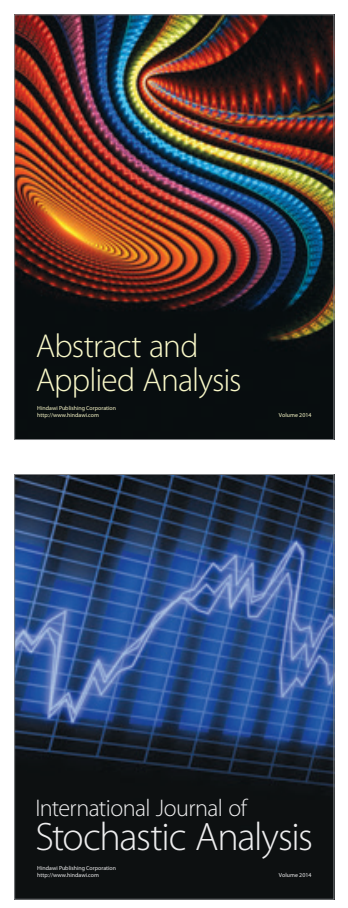

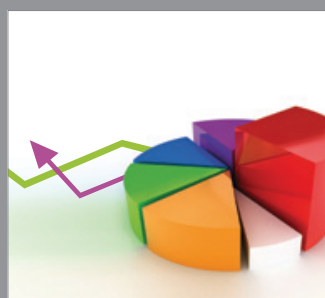

ournal of

Probability and Statistics

Promensencen
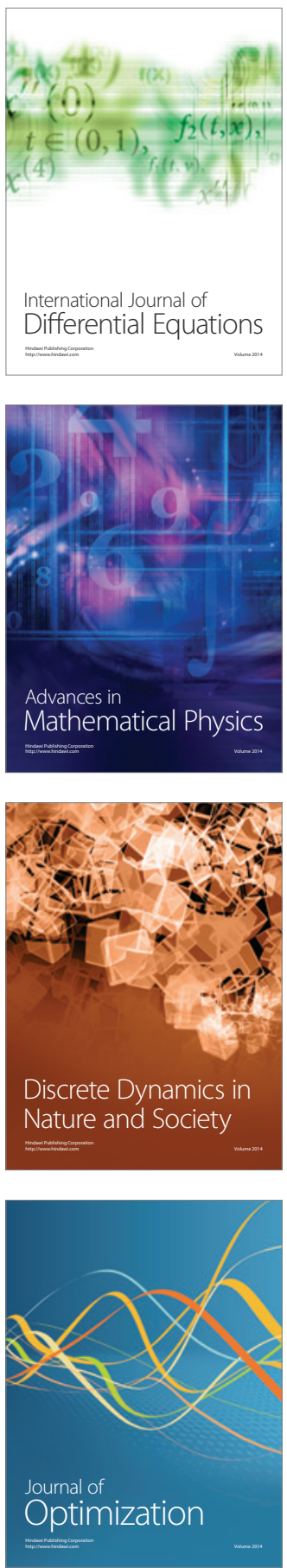